The American Journal of Drug and Alcohol Abuse, 31:571-591, 2005

Copyright (C) Taylor \& Francis Inc.

ISSN: 0095-2990 print / 1097-9891 online

DOI: $10.1081 / A D A-200068120$

\title{
Cognitive Performance by Humans During a Smoked Cocaine Binge-Abstinence Cycle
}

\author{
Edward F. Pace-Schott, ${ }^{1}$ Robert Stickgold, ${ }^{1}$ Amir Muzur, ${ }^{1}$ \\ Pia E. Wigren, ${ }^{2}$ Amie S. Ward, ${ }^{2}$ Carl L. Hart, ${ }^{2}$ \\ Matthew Walker, ${ }^{1}$ Chris Edgar, ${ }^{3}$ and J. Allan Hobson ${ }^{1}$ \\ ${ }^{1}$ Center for Sleep and Cognition, Department of Psychiatry, Harvard Medical \\ School, Beth Israel Deaconess Medical Center, Boston, Massachusetts, USA \\ ${ }^{2}$ Substance Use Research Center, Department of Psychiatry, Columbia University \\ College of Physicians and Surgeons, New York, New York, USA \\ ${ }^{3}$ Cognitive Drug Research, Ltd., Oxon, United Kingdom
}

\begin{abstract}
Five cocaine-dependent individuals completed a 22-day inpatient study of sleep and cognition. Following 3 days of drug-free baseline, participants underwent 3 days of twice-daily smoked cocaine base self-administration (650-mg doses, 14 minutes apart), followed by 15 days of abstinence. Each morning and afternoon, the CDR repeatable, multiple-version, computerized cognitive battery (whose stability following practice has been documented) was administered. During abstinence, performance deteriorated on vigilance tasks (especially reaction time) as well as on immediate and delayed verbal recognition tasks but not on working memory tasks. Declines were most evident in the afternoon. Data suggest that abstinence can unmask cognitive deficits induced by chronic cocaine use and circadian factors may mediate their severity.
\end{abstract}

Keywords: Cocaine, abstinence, binge, cognition, attention, memory, repeated assessments

This research is dedicated to the memory of Dr. Marian W. Fischman, who died before the completion of this study. Dr. Fischman's guidance on the design of this study was invaluable and she will be missed immensely. The authors thank the National Institute on Drug Abuse for supporting this research with DA11744; and Cognitive Drug Research, Ltd. for providing and analyzing the CDR battery. Chris Edgar is an employee of Cognitive Drug Research, Ltd.

Address correspondence to Edward F. Pace-Schott, Beth Israel Deaconess Medical Center, Feldberg 864, 330 Brookline Ave., Boston, MA 02215, USA; Fax: (617) 667-8498; E-mail: epacesch@bidmc.harvard.edu 


\section{INTRODUCTION}

Much concern has been raised recently regarding possible adverse effects of cocaine on cognitive functioning (1-9). In general, these studies have compared abstinent cocaine abusers to matched controls with or without a history of other substance use disorders (6). Several of these studies have documented poorer performance in abstinent cocaine users on measures of elemental cognitive functions including attention, vigilance, reaction time, psychomotor speed, and spatial and verbal memory $(1,3,6-8,10-15)$. In abstinent cocaine users, greater residual neurocognitive impairment is correlated with greater duration, frequency, peak dose and total amount of cocaine consumed per unit time (1-3).

Although there is much agreement about the presence of heterogeneous neurocognitive impairments in many chronic cocaine users compared to controls, findings for a particular cognitive domain or on specific neuropsychological tests have not been consistent among studies [for a review of attentional findings see Horner (6)]. Inconsistencies occur even among well-designed studies with close monitoring of abstinence, strict inclusion and exclusion criteria, as well as scrupulous, multidimensional matching of controls (6). One source of heterogeneity is variability in time elapsed from cessation of use to the point of neurocognitive assessment (6). While repeated measures can address this variability, previous test-retest intervals have been chosen primarily to detect signs of long-term recovery from putative neurotoxicity associated with cocaine abuse [e.g., Refs. $(3,10)]$, rather than the naturalistic variability of cognitive integrity seen in cycles of drug use and abstinence. Therefore, existing longitudinal studies of cognition in cocaine abstinence have not addressed possible changes occurring during the days immediately following drug cessation. Moreover, Horner (6) notes that the necessary duration of abstinence before cognitive function stabilizes is currently unknown.

To address these issues, we have used a laboratory model of cocaine binge use and abstinence (16-18) together with a repeatable, multipleversion computerized cognitive battery, the Cognitive Drug Research, Ltd. (CDR) Battery (see Methods) that shows documented stability following practice (19-25). We report here the first longitudinal, twice-daily assessment of cognitive functioning in chronic cocaine users under controlled, inpatient conditions that include laboratory administration of cocaine followed by 2 weeks of confirmed abstinence.

\section{METHODS}

\section{Participants}

Five research participants (4 males: 3 Black, 1 White; 1 female: Black) 3041 years in age (mean $=35.4, \mathrm{SD}=4.4)$ were solicited through word-of-mouth 
referral and newspaper advertisements in New York, NY. All were current smoked cocaine users and none expressed a desire for treatment of cocaine abuse or dependence. Participants reported using cocaine an average of 37 days per week (mean $4.7 \pm 1.7$ days), spending an average of $\$ 150$ to $\$ 400$ on cocaine per week (mean $\$ 310 \pm \$ 96$ per week), and having used cocaine for $10-20$ years (mean $16.3 \pm 3.8$ years). Street cocaine in the New York City area at the time of the study was $\$ 25$ to $\$ 40$ (mean $\$ 32.5$ ) per gram. All reported using cocaine in a "binge" pattern. Three participants reported current alcohol use (2-10 drinks per week), 2 reported current marijuana use (1-2 times per week), and all smoked 1-20 tobacco cigarettes per day. Other reported drug use was infrequent. The participants had completed 9 to 16 years of education. All participants passed medical and psychological evaluation prior to the study, and none were receiving psychiatric treatment. Each participant signed a consent form approved by the Institutional Review Boards of the College of Physicians and Surgeons of Columbia University and The New York State Psychiatric Institute.

\section{Protocol}

Participants completed a 22-day inpatient study protocol at the Clinical Research Center (GCRC) of the Columbia-Presbyterian Medical Center under the direction of the Columbia College of Physicians and Surgeons Substance Use Research Center (SURC). The study consisted of 3 days initial abstinence ("washout" phase), 3 days of twice-daily smoked cocaine selfadministration ("binge" phase), and 15 days abstinence ("abstinence" phase). Participants were admitted on a Friday (Day 1) and were screened for sleep disorders the following night (Day 2) using polysomnography (PSG) (26). Only one participant was studied at a time.

Participants went to bed at the same time each night and were awakened at the same time each morning. Bedtime was identical for all participants and was chosen so as to give participants the opportunity to sleep up to 8.25 hours per night.

Prior to enrollment, participants were informed that they would not be allowed to nap. Both research nurses and unit nurses performed hourly checks to enforce compliance with this instruction. Every evening, participants reported any napping during the preceding day on a questionnaire. Based upon self-report, 3 participants were fully compliant with this instruction; Only 2 reported napping-one on 8 of the 15 abstinence days and the other on 3. Daily reported nap times increased over abstinence in the former participant but not in the latter. Participants were allowed to watch television, listen to music, and engage in other recreational activities during daytime hours and they were provided with a television, VCR, stereo, and telephone in their room. 


\section{Cocaine Sessions}

During experimental sessions, each participant was seated in a reclining lounge chair in front of a computer monitor. The electrocardiogram was continuously monitored via chest electrodes (Tektronix 413 Monitor, Beaverton, OR; MAC PC, Marquette Electronics, Milwaukee, WI), while heart rate (HR) and blood pressure (systolic, SP; diastolic, DP) were recorded every 2 minutes (Sentry II-Model 6100 automated vital signs monitor, NBS Medical, Costa Mesa, CA) beginning 20 minutes prior to drug administration. An Apple Macintosh IIci computer located in an adjacent room was used for automated data collection.

Experimental sessions were conducted at approximately 11:00 and 14:00, and began with 30 minutes of baseline measurement of cardiovascular activity and mood. Cocaine base (50 mg smoked) was administered up to 6 times in each of the 2 daily sessions with periods of availability within a session occurring every 14 minutes. A metered dose of cocaine base was placed in a glass tube $(8 \mathrm{~cm}$ in length) packed with fine metal mesh. Participants held this tube while the research nurse held the flame from a lighter until the individual had finished inhaling the smoke.

Dose availability was signaled by the appearance in outline form of 2 squares $(3 \mathrm{~cm} \times 3 \mathrm{~cm})$ on the computer monitor. Participants selected or rejected the 50-mg dose by moving the cursor to the left or right (illuminating the square associated with that position) with one side associated with accepting and the other with rejecting the dose. Participants then pressed a button on the mouse to select that choice. Participants invariably accepted cocaine doses. Cocaine was not given on any trial in which cardiovascular activity was above our criteria for safe drug administration $(\mathrm{HR}<130$, $\mathrm{DP}<100, \mathrm{SP}<165)$.

The cocaine self-administration procedure in this study, wherein participants simply accept or reject a dose, should be differentiated from participant-directed human self-administration procedures used in some other experimental settings. For example, one typical SURC cocaine selfadministration protocol consists of one sample trial, where participants receive the cocaine dose and the alternative reinforcer available that day, followed by 5 choice trials, where participants choose between the available cocaine dose and an alternative reinforcer.

\section{Drug}

Cocaine hydrochloride, provided by the National Institute on Drug Abuse, was heated with sodium bicarbonate and water to $93^{\circ} \mathrm{C}$, and subsequently cooled, dried, and weighed to form cocaine free base by the ColumbiaPresbyterian Medical Center Research Pharmacy (27). 


\section{Cognitive Testing}

A repeatable battery of computerized tasks (CDR battery; Cognitive Drug Research, Ltd., Goring-on-Thames, Oxon, UK, www.cdr.org.uk), was used to measure participants' cognitive functioning on the morning (AM tests) and evening (PM tests) of every protocol day except abstinence Days 9-10 and 16-17. (Personnel constraints necessitated omission of CDR sessions during the two weekends of the protocol.) A consistent temporal separation between $\mathrm{AM}$ and PM testing was maintained and is detailed in the Results section.

Each CDR battery component task exists in over 40 equivalent alternate versions that are presented to each participant in a randomized order. No single variant was presented more than once to a given participant over the course of a given study. Participants completed a total of 32 testing sessions (i.e., 32 variants of each component task) over the course of the 21-day study.

The multiple versions of the CDR tasks have been demonstrated to produce stable performance in normal participants after 4 practice sessions $(21,23,25)$. Results from the first 7 sessions, all occurring during the initial washout period, were excluded from analyses.

The CDR task battery consists of the following 7 tests more details about which may be found in Wesnes et al. (19).

In the Simple Reaction Time (SRT) test, participants responded by pressing the "yes" button as quickly as possible when the word "yes" appears, and reaction time (SRT) was recorded.

In the Choice Reaction Time (CRT) test, participants responded by pressing the "yes" or "no" button, as quickly as possible, when the word "yes" or "no" appears. Both CRT reaction time (CRT_RT) and CRT accuracy (CRT_Acc) were recorded.

In the Digit Vigilance (DV) task, a single digit was displayed on one side of the screen while single digits were presented in rapid succession in the middle of the screen. Participants pressed the "yes" button whenever a digit matched the one displayed to the side. Both reaction time (DV_RT) and accuracy (DV_Acc) were recorded.

In the word recognition and working memory tasks, participants were instructed to respond as quickly and as accurately as possible but to guess if unsure.

For the Immediate (IR) and Delayed Word Recognition (DR) tasks, participants first viewed a series of 15 words appearing one at a time on the screen and were instructed to remember them. Immediately after viewing these stimuli (IR), or after an approximately 20-minute delay (DR) during which other CDR tasks were performed, participants were shown a series of new and old words, and pressed the "yes" button if the word had been viewed before (original stimuli), or "no" if it had not (new stimuli). The percentages of original $(\mathrm{O})$ and new $(\mathrm{N})$ words accurately identified (Acc) were calculated (IR_or DR_OAcc, and IR_or DR_NAcc, respectively). 
In the Spatial Working Memory (SWM) task, participants first viewed a house with 9 windows, 4 of which were lit. The participant then viewed a series of pictures of the house with only one window lit, and pressed "yes" if that window was lit in the original display or "no" if it was not. The percentage of originally lit windows (SWM_OAcc) and previously unlit windows (SWM_NAcc) accurately identified as such were calculated.

In the Numeric Working Memory (NWM) task, participants viewed 5 numbers, one at a time, and were instructed to remember them. The participants were then shown a series of numbers and pressed "yes" for previously viewed numbers and "no" for new numbers. The percentage of original (NWM_OAcc) and new (NWM_NAcc) numbers accurately identified as such were calculated.

One canonical variable, Power of Attention (POA), and one theoretically derived variable, Processing Time (PRO_TM), were also calculated. Power of Attention combined 3 measures of vigilance reaction time and was equal to the sum of SRT, CRT_RT, and DV_RT. Processing time was the theoretical duration of cognitive processing involved in choosing one or the other choice on the CRT, separate from psychomotor response speed, and was the difference between CRT_RT and SRT.

\section{Analyses}

The study had a within-participants, repeated measures design that allows significant effects to be measured despite low sample size. Cognitive measures were analyzed using simple regression, repeated measures ANOVA, and the nonparametric Friedman Test.

Performance Changes Over Course of Abstinence

Simple Regression. The 5-participant means were calculated for each of the above cognitive variables for each day of abstinence (Days 7-21 excepting nontesting Days 9, 10, 16, and 17). AM and PM test sessions means were analyzed separately.

Friedman Test. Given the small sample size, AM and PM test variables showing significance or trends with simple regression were re-examined using the nonparametric Friedman test corrected for ties (28). Because this test does not allow the number of experimental days to exceed the number of participants, 5 evenly spaced abstinence days (Days 7, 11, 14, 18, and 21, corresponding to the 1st, 5th, 8th, 12th, and 15th day of abstinence) were analyzed.

\section{Performance Differences Between Binge and Abstinence}

Separate AM and PM means for each test variable were calculated for each participant across binge phase days (defined as Days 5-7 for AM tests and 
Days 4-6 for PM tests) and across the last week abstinence (Days 15 and 18-21). Data from the last week of abstinence were selected to ensure that all abstinence data were collected well beyond any acute sequelae of cocaine self-administration. Repeated measures ANOVAs were then carried out separately for AM and PM test sessions to assess main effects of the repeated measure (Phase).

Time-of-Day Main Effects and Interactions with Study Phase and Day-of-Abstinence

To investigate the main effect of Time-of-Day (AM vs. PM) and interactions of Time-of-Day with experimental phase or duration of abstinence, 2 nested repeated measures ANOVAs were performed on each CDR variable. In the first, the superordinate factor was Phase which had two levels-binge and abstinence (with each participant's phase means for each variable computed as above). In the second, the superordinate factor was Day-of-Abstinence which had 11 levels-ie, the 11 abstinence days on which participants were tested. In both cases, Time-of-Day (AM versus PM) was the subordinate nested factor.

\section{RESULTS}

\section{Cocaine Consumed}

No participant ever refused a cocaine dose and, therefore, participants consumed the full amount of cocaine made available for a total estimated intake of $300 \mathrm{mg} / \mathrm{session}, 600 \mathrm{mg} / \mathrm{day}$, and $1800 \mathrm{mg} / 3$-day simulated binge. This amount was, however, less than the average amount these participants consumed in the natural setting (approximately 9.54 grams per week, or $4,080 \mathrm{mg} /$ three days as estimated using mean cost and expenditure data reported in the Methods section).

\section{Testing Times}

During abstinence (Days 7-21), morning (AM) testing began at a (military time) mean of $8: 37(\mathrm{SD}=0: 55)$ with individual participant means (and SDs) of 8:07 (1:45), 8:41 (1:03), 8:12 (1:45), 9:29 (0:23) and 8:29 (1:15). Afternoon (PM) testing began at a mean of $15: 19(\mathrm{SD}=0: 16)$ with individual participant means (SD's) of 15:11 (1:03), 15:25 (0:34), 15:21 (0:17), 15:16 $(0: 35)$, and 15:22 (0:28). Although daily variation in GCRC demands on unit staff resulted in variance of $\mathrm{AM}$ and $\mathrm{PM}$ abstinence testing times, especially in AM, simple regression showed that neither mean AM or PM testing times, nor the duration between tests $(6.68 \pm 1.13 \mathrm{hr})$ varied systematically with experimental day (Figure 1). 


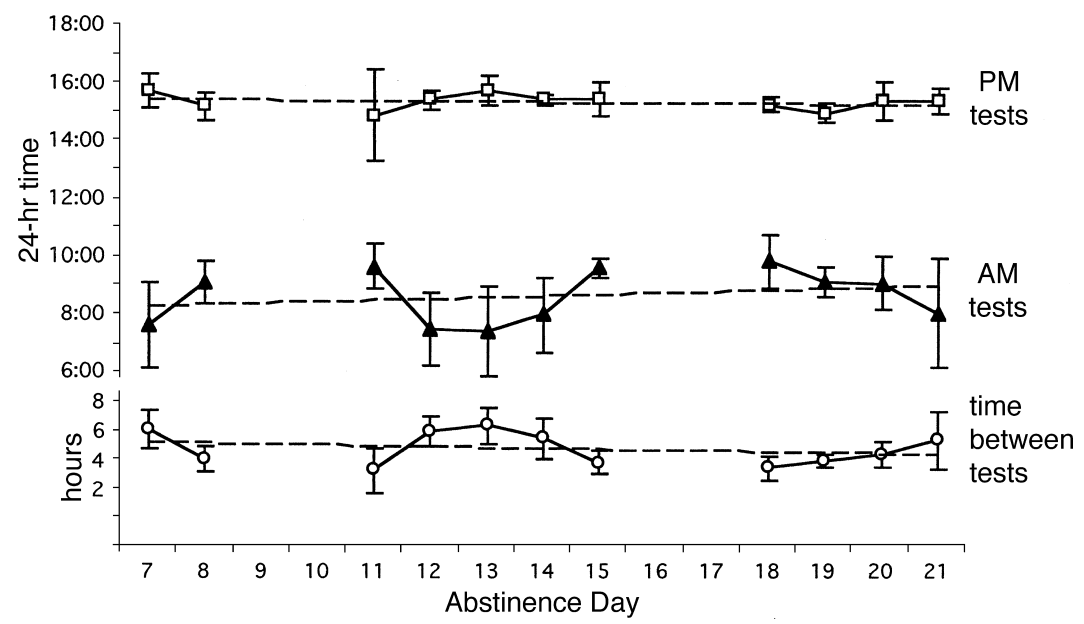

Figure 1. Morning (AM) and afternoon (PM) cognitive testing times (in military time) over abstinence period. Upper trace PM test sessions; middle trace AM test sessions; bottom trace time between test sessions. Y-axes: upper and middle trace, clock time; lower trace, time in hours. Bars are standard deviation. Dotted lines are simple regression lines computed for time against day of abstinence none of which are significant.

On binge days (Days 4-6), the test batteries were given prior to their first (AM) cocaine self-administration session (average $8: 12, \mathrm{SD}=0: 39$ hour) and following their PM session (average $=16: 28, \mathrm{SD}=0: 06$ ) with a mean intertest time of $8.25 \pm 0.60$ hour. Mean AM test times during binge did not differ from those during abstinence, but mean PM test times during binge were significantly later than those during abstinence (mean difference $=1.15$ hours) and mean intertest intervals were significantly longer during binge (mean difference $=1.53$ hours).

\section{Performance Changes Over Course of Abstinence}

\section{Simple Reaction Time}

Using simple regression, SRT increased significantly across abstinence for both AM and PM tests (AM, p<.05, r =.62; PM, p<.05, r = .68, Figure 2a). The slowing of mean SRT from the first to last day of abstinence was $36 \%$ for the AM tests and $31 \%$ for PM tests. The Friedman test showed a trend for PM SRT to differ with days $(\mathrm{p}=.08$, mean rank order Days $7<11<14<18<21$ ) but not for AM SRT.

\section{Choice Reaction Time}

Simple regression analysis showed that mean CRT reaction time (CRT_RT) increased $(\mathrm{p}<.05, \mathrm{r}=.69)$ and CRT accuracy declined $(\mathrm{p}<.05, \mathrm{r}=.71)$ 
a.

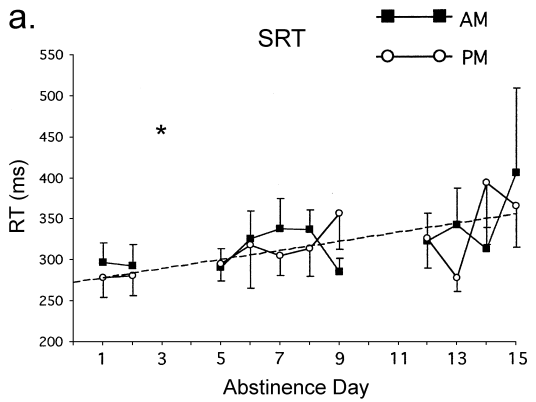

C.

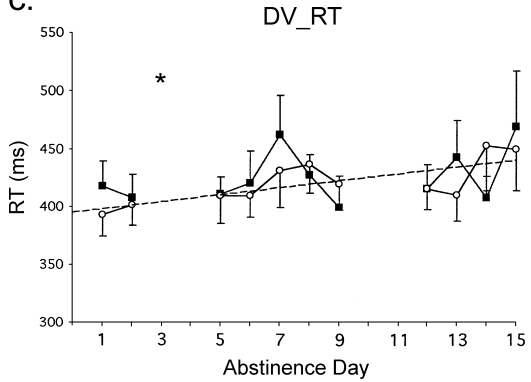

b.

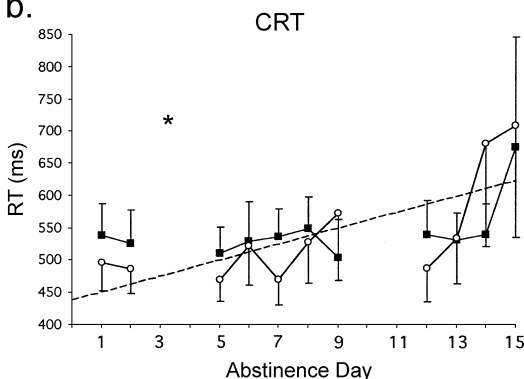

d.

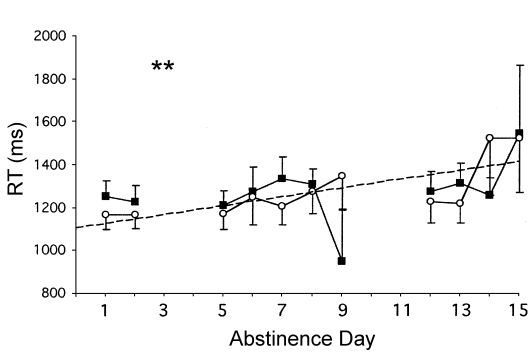

Figure 2. Changes in reaction time (in ms) across 15 days of abstinence on the three CDR vigilance tests and their sum at AM and PM test sessions. Darkened squares and upgoing standard error bars are AM tests while open circles and downgoing standard error bars are PM tests. The dotted line is the simple regression line computed for PM data as a function of abstinence day. Data points are mean reaction time with standard error for the 5 participants on each abstinence day. (a) Simple reaction time (SRT). (b) Choice reaction time (CRT). (c) Digit vigilance reaction time (DV_RT). (d) Power of attention (POA), the sum of SRT, CRT and DV_RT. Asterisk (*), regression of PM data significant at $\mathrm{p}<.05 ; * *$ significant at $\mathrm{p}<.01$.

significantly across abstinence in PM but not in AM tests (Figure 2b). From the first to last day of abstinence, mean PM reaction time slowed by $42 \%$, while error rate increased by $167 \%$. Friedman tests showed a trend for PM CRT_RT to differ with days $(\mathrm{p}=.09$, mean rank order Days $11<18<7<14$ and 21$)$.

\section{Processing Time}

Simple regression showed a trend toward increasing Processing Time (choice RT minus simple RT) in PM tests $(\mathrm{p}=.06, \mathrm{r}=.59)$ but not AM tests. Friedman tests showed no significant difference across abstinence days.

\section{Digit Vigilance}

Simple regression showed that mean DV reaction time (DV_RT) increased significantly across abstinence in PM $(\mathrm{p}<.05, \mathrm{r}=.73)$ but not AM tests 
(Figure 2c). Friedman tests showed significant difference among days for PM DV_RT $(\mathrm{p}<.05$, mean rank order Days $7<11<18<14<21)$ but not for AM values. No changes in DV accuracy were seen.

\section{Power of Attention}

Simple regression showed that POA, a sum of 3 constituent RT measures (simple RT+choice RT+digit vigilance RT) increased significantly across abstinence in PM $(\mathrm{p}<.01, \mathrm{r}=.74)$ and was a trend in AM tests $(\mathrm{p}=.09, \mathrm{r}=.53$; Figure 2d). Friedman tests for AM and PM POA did not reach trend or significance although the PM mean rank order was similar to that of its constituent RTs (Days $7<11<18<14<21$ ).

\section{Immediate Word Recognition}

Simple regression showed that mean IR original stimuli accuracy (IR_OAcc) significantly declined across abstinence in PM $(\mathrm{p}<.01, \mathrm{r}=.82)$ but not AM tests (Figure 3a). Friedman tests showed significant difference among days for PM IR_OAcc $(\mathrm{p}<.05$, mean rank order Days $21<18<11<14<7)$ but not for AM IR_OAcc. There was no change across abstinence in IR new word accuracy.

a.

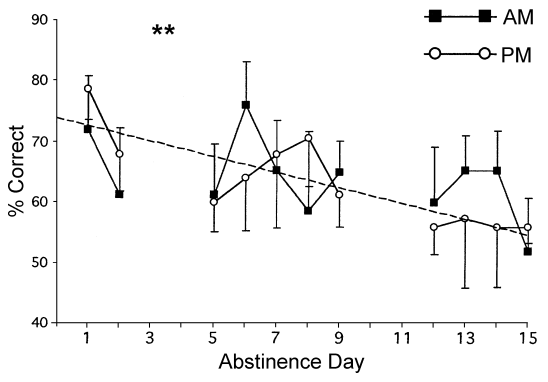

b.

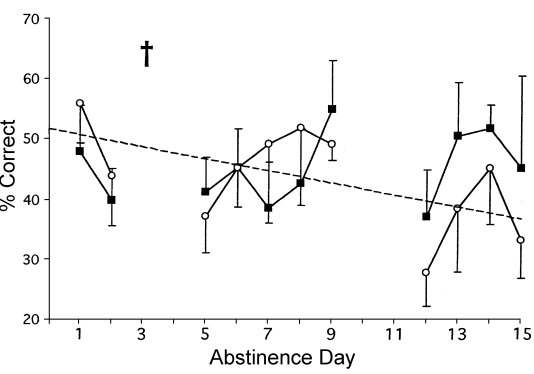

Figure 3. Changes in CDR immediate (IR) and delayed (DR) percent accuracy in identifying previously seen ("original") word stimuli (OAcc) across 15 days of abstinence. Darkened squares are AM tests while open circles are PM tests. The dotted line is the simple regression line computed for PM data as a function of abstinence day. Data points are mean percent accuracy with standard error for the 5 participants on each abstinence day. (a) Immediate Word Recognition Accuracy (IR_OAcc). (b) Delayed Word Recognition Accuracy (DR_OAcc). Asterisk (**), significant at $\mathrm{p}<.01 ; \dagger$, a trend at $\mathrm{p}<.1$. 
Delayed Word Recognition

Simple regression showed that mean DR original stimuli accuracy (DR_OAcc) showed a trend toward decrease across abstinence in PM $(\mathrm{p}=.08, \mathrm{r}=-.56)$ but not AM tests (Figure $3 \mathrm{~b})$. Friedman tests showed a trend for difference among days for PM DR_OAcc ( $\mathrm{p}=.05$, mean rank order Days $18<21<11<14<7$ ) but not for AM DR_OAcc. There was no change across abstinence for DR new stimuli accuracy.

Spatial and Numeric Working Memory

Simple regression showed no changes across abstinence in AM or PM SWM original stimuli accuracy (SWM_OAcc). Friedman tests showed a significant difference among days for PM SWM_OAcc ( $\mathrm{p}<.05$, mean rank order Days 7 and $14<11<21<18$ ) but not for AM SWM_OAcc. There were no changes across abstinence for SWM new stimuli accuracy or for any NWM task variable.

\section{Performance Differences Between Binge and Last Week of Abstinence}

\section{Simple Reaction Time}

ANOVA showed that the increase in mean SRT between binge and the last week of abstinence did not reach significance or a trend in AM or PM tests. However, the effect size was large for PM tests (.90) suggesting that, with a larger sample size, there would be sufficient statistical power to achieve significance (29).

\section{Choice Reaction Time}

ANOVA showed a trend for mean PM CRT accuracy (CRT_Acc) to decline between binge and the last week of abstinence, $F(1,4)=4.6, p=.10$ (effect size $-.65)$, but no differences for AM CRT_Acc. There were no binge-abstinence differences for CRT reaction time.

Processing Time

There were no binge-abstinence differences for Processing Time.

Digit Vigilance

ANOVA showed a significant increase in mean PM DV reaction time (DV_RT) between binge and the last week of abstinence, $\mathrm{F}(1,4)=9.9, \mathrm{p}<.05$ 
(effect size=.96), but not in AM DV_RT. DV accuracy did not differ between binge and abstinence.

\section{Power of Attention}

ANOVA showed an trend for PM POA to increase between binge and the last week of abstinence, $F(1,4)=5.6, p=.08$ (effect size $=.95)$, but no difference for AM POA.

Immediate Word Recognition

ANOVA showed a significant decrease in mean PM IR original word accuracy (IR_OAcc) between binge and the last week of abstinence, $\mathrm{F}(1,4)=9.7, \mathrm{p}<.05$ (effect size $=-2.01$ ), and no change, but a large effect size $(-.97)$, for AM IR_OAcc. There were no binge-abstinence differences for IR new word accuracy.

Delayed Word Recognition

ANOVA showed a trend for mean PM DR original word accuracy (DR_OAcc) to decline between binge and the last week of abstinence, $\mathrm{F}(1,4)=4.8, \mathrm{p}=.09$ (effect size $=-1.46$ ), but no difference for AM DR_OAcc. There were no binge-abstinence differences for DR new word accuracy.

Spatial and Numeric Working Memory

ANOVA showed an increasing trend of PM SWM original stimuli accuracy (SWM_OAcc), $\mathrm{F}(1,4)=4.5, \mathrm{p}=.1$ (effect size=.9) between binge and the last week of abstinence, but no change in AM SWM_OAcc. There were no binge-abstinence differences for SWM new stimuli accuracy or in any NWM task variables.

\section{Time-of-Day Main Effects and Interactions with Study Phase and Day-of-Abstinence}

There were few main effects for Time-of-Day or interactions between Timeof-Day and Phase using ANOVA with Time-of-Day nested below Phase (i.e., binge or abstinence). There was a significant Time-of-Day main effect for Digit Vigilance RT (DV_RT), $F(1,4)=8.8, \mathrm{p}<.05$, with mean reaction time slower in the morning. There was a similar trend toward slower AM than PM mean reaction time as measured by Power of Attention (the sum of the three vigilance $\mathrm{RTs}$ ), $\mathrm{F}(1,4)=4.8, \mathrm{p}=.09$. Time-of-Day $\times$ Phase interaction was significant for Spatial Working Memory original stimuli accuracy, 
$\mathrm{F}(1,4)=10.1, \mathrm{p}<.05$, and was a trend for DV_RT, $\mathrm{F}(1,4)=6.5, \mathrm{p}=.06$. In both cases, Time-of-Day differences were greater during binge phase.

There were still fewer main effects for Time-of-Day or interactions between Time-of-Day and Day-of-Abstinence using ANOVA with Time-ofDay nested below Day-of-Abstinence. Only DV_RT approached significance, $\mathrm{F}(1,4)=4.7, \mathrm{p}=.1$, again with slower AM RT. The only Time-of-Day $\times$ Dayof-Abstinence interaction approaching significance was for Simple Reaction Time, $\mathrm{F}(10,40)=1.8 . \mathrm{p}=.1$.

\section{DISCUSSION}

\section{Performance Changes Across the Binge-Abstinence Cycle}

This is the first study, in chronic cocaine users, of day-to-day changes in elemental cognitive abilities during confirmed abstinence under controlled inpatient conditions and after administration and withdrawal from cocaine at precisely known times. It is also the first to assess time-of-day effects on cognitive performance in abstinent cocaine users.

Results indicate that 2 weeks of confirmed abstinence are accompanied by performance declines in attention and recognition memory but not in working memory which may, in contrast, improve slightly in the spatial domain. Attentional declines over abstinence were indicated by increasing reaction times on vigilance tasks. Recognition memory performance declines over abstinence appeared as decreasing accuracy at identifying previously seen stimuli. Declining performance across abstinence was evident in midafternoon testing sessions but not in testing sessions administered shortly after morning awakening. These results were evident using both parametric and non-parametric measures. The same attention and recognition memory variables that showed worsening performance over abstinence days also worsened from their overall mean during binge to their overall mean during the last week of abstinence, and this deterioration also was evident in afternoon but not morning test sessions.

The time-of-day difference in performance changes over abstinence was, however, superimposed on quite similar absolute AM and PM performance values. Figures 2 and 3, show that both $\mathrm{AM}$ and PM daily 5-participant means of each variable remained near the regression lines computed for PM means alone. The main difference between AM and PM performance appears to be poorer AM performance early in abstinence, leading to less overall change in AM performance across the abstinence period. It is, therefore, not surprising that abstinence grand means of AM and PM scores were similar and that there were few Time-of-Day main effects or interactions with Phase (binge and abstinence) or Day-of-Abstinence (11 successive days). The 2 Time-of-Day $\times$ Phase interactions appear driven by greater differences between $\mathrm{AM}$ and $\mathrm{PM}$ during the binge phase when PM testing was 
confounded by cocaine use a short time earlier. In one case, binge phase PM digit vigilance reaction time was faster in the PM than in the AM (with no corresponding reduction in digit vigilance accuracy). In the other case, binge phase PM spatial working memory accuracy was poorer in the PM than in the AM.

\section{Comparison of Vigilance RT Changes with Other Studies}

Reviewing well-controlled studies in which confirmed abstinent cocaine users were studied after at least several weeks abstinence [e.g., Refs. $(10,11$, 30)], Horner (6) concluded that tasks assessing information processing speed such as the PASAT show the most consistent pattern of impairment relative to controls. Among such tasks, Horner (6) includes the divided attention reaction time tasks used by Bauer (10) whose most difficult versions produced reaction times near $500 \mathrm{~ms}$ in cocaine users abstinent 7-100 days. Such RTs are similar to those seen in the last week of abstinence for our choice reaction time and digit vigilance tasks (Figure 2).

Because his abstinent cocaine users were equally impaired in reaction time on auditory and visual tasks, Bauer (10) suggested that motor rather than perceptual components of reaction times may be the source of impairment in abstinent cocaine users. An alternative explanation in the current study is that participants increased their reaction time in order to preserve accuracy, an explanation consistent with the trend toward increasing processing time over abstinence in PM tests. However, performance declines also were seen in simple reaction time, a test that presumably requires only a basic perceptualmotor response.

Di Sclafani et al. (3) found that reaction time was one of few subtests on the MicroCog battery unimpaired relative to normals in cocaine users abstinent 6 weeks to 6 months. However, although recovery of vigilance RT capacity may take place with longer periods of abstinence, Bauer (10) found no group differences in reaction time between cocaine users abstinent 7-10, 16-21, and 94-100 days. Based on inconsistent findings such as these, Horner (6) concluded that there was insufficient evidence to state unequivocally that attentional dysfunction accompanies cocaine abstinence. To address such inconsistencies, future studies on the time course of abstinence-related vigilance declines among different populations of chronic cocaine users should strive toward standardizing the specific tasks employed across studies as well as performing frequent repeat testing throughout medium and long-term abstinence.

Our findings demonstrate that, at least across the first 2 weeks, duration of abstinence contributes significantly to changes in vigilance performance. Furthermore, our findings suggest that long-term reaction time slowing relative to normals, when observed, begins during the first few weeks of abstinence. Lastly, our findings suggest that the time of day at which testing 
occurs also requires strict control especially when following performance across the early days of abstinence.

\section{Comparison of Verbal Memory Changes with Other Studies}

Although verbal memory recall in abstinent cocaine users has been reported to be deficient $(31-33)$, verbal recognition memory has received much less attention. Bondi et al. (31) noted that while abstinent polysubstance abusing heavy cocaine users scored more poorly on verbal recall portions of the California Verbal Learning Test than those with a less severe cocaine history, this difference was not seen for recognition memory (Recognition Discriminability). Notably, however, Easton and Bauer (34) found that in abstinent cocaine users selected for evidence of memory impairment, oral thiamine improved their performance on a recognition memory task using letter stimuli (Sternberg Memory Scanning), a finding consistent with the presence of a pre-oral thiamine deficit.

\section{Correlates of Cognitive Changes}

Repeated assessments of cognitive integrity during actual drug use followed by confirmed abstinence allow us to generate hypotheses on possible correlates of observed cognitive fluctuations. Such correlates include circadian effects on performance, abstinence-related changes in sleep quality, and neuromodulatory changes.

Time-of-day differences in day-to-day performance declines were present over successive abstinence days when, unlike during binge, such differences cannot be explained by intervening drug use. The PM tests occurred in temporal proximity to a putatively endogenous mid-afternoon increase in sleepiness (35) which might exacerbate duration-of-abstinence effects on vigilance. The same effect might be expected if the time-awake related accumulation of homeostatic sleep pressure was increasingly exacerbated by poor sleep over successive days of cocaine abstinence. In contrast, AM tests occurred during a circadian period characterized by increasing CNS activation thereby potentially ameliorating these abstinence effects.

Reciprocal interaction of cocaine use and circadian rhythms have been documented. Distinct circadian patterns of cocaine self-administration occur in rats (36) and perhaps also in humans (37) while chronic cocaine can alter circadian rhythms in rats (38). In human cocaine users, circadian fluctuation in cognitive integrity may play a mediating role in patterns of substance use while cocaine use may, in turn, exacerbate low points in normal circadian patterns of alertness.

Observed cognitive declines were temporally associated with sleep quality declines in these same participants (26). Across the binge-abstinence 
sequence, mean core sleep quality variables (sleep duration, efficiency and latency) changed significantly in the direction of poorer sleep replicating previous inpatient studies $(39,40)$. Sleep effects on cognition are increasingly documented in the domains of vigilance, attention and psychomotor performance [e.g., Ref. (41)], executive function [e.g., Ref. (42)] and learning and memory [e.g., Ref. (43)]. However, identification of causal relationships between sleep and cognitive variables in chronic cocaine users must await experimental manipulations that dissociate both impairments from temporally covarying factors.

The current findings are consistent with the hypothesis that chronic cocaine use degrades the neuromodulatory substrates of attention and memory. Enduring effects of chronic cocaine use have been documented in dopaminergic [e.g., Ref. (44)], serotonergic [e.g., Ref. (45)], noradrenenrgic [e.g., Ref. (46)], cholinergic [e.g., Ref. (47)], glutamatergic [e.g., Ref. (48)], and GABAergic [e.g., Ref. (49)] systems. For example, down-regulation of striatal postsynaptic dopamine D2 receptors (50) and alteration of serotonergic systems (51) accompany human chronic cocaine abuse. Although actual cocaine-induced degeneration of neuromodulatory neurons appears unlikely [e.g., Refs. $(46,52,53)]$ chronic alterations in receptor sensitivity or transporter function could directly or indirectly affect cognitive functioning.

\section{Interpretive Issues in the Current Study}

Interpretation of these findings must be made in the context of limitations in the current study. Chief among these is the small sample size which limits generalization of these findings to the larger populations of cocaine users. Nonetheless, the statistical power provided by a within-participant repeated measures design allows us confidence in the trends observed from this limited sample. Importantly, while participants' performance on certain tasks declined over abstinence (i.e., vigilance RT and recognition memory tests given in the afternoon), performance on others did not change over abstinence (i.e., Numeric Working Memory and most tests when administered in the morning), or even improved over abstinence (Spatial Working Memory). These findings suggest that the observed performance declines were not simply a nonspecific (e.g., motivational) effect of prolonged hospitalization. Further confidence is indicated by the observation that functions we found to be most labile with increasing duration of abstinencevigilance RT and verbal memory-are also functions which have been shown to differentiate abstinent cocaine users from normal controls [e.g., Refs. $(3,6,54)]$.

Of concern also is the fact that cognitive performance over repeated assessments may be affected by fatigue, motivation and interference (retroactive or proactive) as well as by circadian rhythms. However, prior 
studies with the CDR battery suggest that these factors produce daily fluctuations in performance which return to baseline following a night's sleep [e.g., Refs. $(20,22,24,55)]$. Therefore, with the exception of circadian rhythms discussed above, such factors are unlikely to have produced systematic variation in this study. Lastly, any CDR performance effects of the napping reported by two of the five participants would be expected, if present, to counteract deterioration in CDR performance.

\section{CONCLUSIONS}

Chronic cocaine users show performance declines over time in vigilance reaction time and recognition memory from binge to abstinence days and during abstinence itself. Because pretreatment performance on cognitive tasks (e.g., measures of attention and working memory) have been demonstrated to predict cocaine treatment success $(56,57)$, the findings from the current investigation could have important clinical implications.

\section{REFERENCES}

1. Bolla KI, Funderburk FR, Cadet J-L. Differential effects of cocaine and cocaine alcohol on neurocognitive performance. Neurology 2000; 54:2285-2292.

2. Bolla KI, Rothman R, Cadet J-L. Dose related neurobehavioral effects of chronic cocaine use. J Neuropsychiatry Clin Neurosci 1999; 11:361369.

3. Di Sclafani V, Tolou-Shams M, Price LJ, Fein G. Neuropsychological performance of individuals dependent on crack-cocaine, or crackcocaine and alcohol, at 6 weeks and 6 months of abstinence. Drug Alcohol Depend 2002; 66:161-171.

4. Fein G, Di Sclafani V, Meyerhoff D. Prefrontal cortical volume reduction associated with frontal cortex function deficit in 6-week abstinent crackcocaine dependent men. Drug Alcohol Depend 2002; 68:87-93.

5. Goldstein RZ, Volkow ND. Drug addiction and its underlying neurobiological basis: neuroimaging evidence for the involvement of the frontal cortex. Am J Psychiatry 2002; 159:1642-1652.

6. Horner MD. Attentional functioning in abstinent cocaine abusers. Drug Alcohol Depend 1999; 54:19-33.

7. Robinson JE, Heaton RK, O'Malley SS. Neuropsychological functioning in cocaine abusers with and without alcohol dependence. Int Neuropsychol Soc 1999; 5:10-19.

8. Rosselli M, Ardila A, Lubomski M, Murray S, King K. Personality profile and neuropsychological test performance in chronic cocaineabusers. Int J Neurosci 2001; 110:55-72. 
9. Toomey R, Lyons MJ, Eisen SA, Xian H, Chantarujikapong S, Seidman LJ, Faraone SV, Tsuang MT. A twin study of the neuropsychological consequences of stimulant abuse. Arch Gen Psychiatry 2003; 60:303310.

10. Bauer LO. Psychomotor and electroencephalographic sequelae of cocaine dependence. NIDA Res Monogr 1996; 163:66-93.

11. Beatty WW, Katzung VM, Moreland VJ, Nixon SJ. Neuropsychological performance of recently abstinent alcoholic and cocaine abusers. Drug Alcohol Depend 1995; 37:247-253.

12. Bolla KI, Cadet J-L, London ED. The neuropsychiatry of chronic cocaine abuse. J Neuropsychiatry Clin Neurosci 1998; 10:280-289.

13. Hoff AI, Riordan H, Morris L, Cestaro V, Weineke M, Alpert R, Wang GJ, Volkow ND. Effects of crack cocaine on neurocognitive function. Psychiatry Res 1996; 60:167-176.

14. Roberts LA, Bauer LO. Reaction time during cocaine versus alcohol withdrawal: longitudinal measures of visual and auditory suppression. Psychiatry Res 1993; 46:137-229.

15. Rosselli M, Ardila A. Cognitive effects of cocaine and polydrug abuse. J Clin Exp Neuropsychol 1996; 18:122-135.

16. Foltin RW, Fischman MW. A laboratory model of cocaine withdrawal in humans: intravenous cocaine. Exp Clin Psychopharmacol 1997; 5:404-411.

17. Ward AS, Haney M, Fischman MW, Foltin RW. Binge cocaine selfadministration by humans: smoked cocaine. Behav Pharmacol 1997; 8(8):736-744.

18. Ward AS, Haney M, Fischman MW, Foltin RW. Binge cocaine selfadministration in humans: intravenous cocaine. Psychopharmacology (Berl) 1997; 132(4):375-381.

19. Wesnes KA, Ward T, McGinty A, Petrini O. The memory enhancing effects of a Ginkgo biloba/Panax ginseng combination in healthy middle aged volunteers. Psychopharmacology (Berlin) 2000; 152:353361.

20. Wesnes KA, Garratt C, Wickens M, Gudgeon A, Oliver S. Effects of sibutramine alone and with alcohol on cognitive function in healthy volunteers. Br J Clin Pharmacol 2000; 49(2):110-117.

21. Pincock C, Davies G, Wesnes KA. The effects of training on the quality of performance on computerised cognitive tasks. J Psychopharmacol 1997; 11(Supplement):A57.

22. Wesnes K, Lockton A, Rolan P, Stephenson N, Pincock C. Volunteer study of the potential interaction between remacemide $300 \mathrm{mg}$ and alcohol (0.7 g/kg). J Psychopharmacol 1997; 11(Supplement):A59.

23. Wesnes KA, Ward T. Training effects on tests of cognitive function, and the implications for clinical trials. J Psychopharmacol 2000; 14(Supplement):A47.

24. Wesnes K, Beuzen J-N, Taylor N, Wood A. The effects of olanzapine 
and haloperidol on cognitive function in healthy elderly volunteers. J Psychopharmacol 1997; 11(Supplement):A75.

25. Wesnes K, Pincock C. Practice effects on cognitive tasks: a major problem? Lancet Neurol 2002; 1(8):473.

26. Pace-Schott EF, Stickgold R, Muzur A, Wigren P, Ward AS, Hart C, Clarke D, Fischman MW, Hobson JA. Sleep quality deteriorates over a binge-abstinence cycle in chronic smoked cocaine users. Psychopharmacology 2005; 179:873-883.

27. Foltin RW, Fischman MW, Nestadt G, Stromberger H, Cornell EE, Pearlson GD. Demonstration of naturalistic methods for cocaine smoking by human volunteers. Drug Alcohol Depend 1990; 26(2):145-154.

28. Zar JH. Biostatistical Analysis. 2nd ed. Englewood Cliffs: Prentice Hall, 1984.

29. Lipsey MW, Wilson DB. Practical Meta-Analysis. Thousand Oaks, CA: Sage Publications, 2000.

30. Bauer LO. Vigilance in recovering cocaine-dependent and alcoholdependent patients: a prospective study. Addict Behav 1994; 19:599607.

31. Bondi MW, Drake AI, Grant I. Verbal learning and memory in alcohol abusers and polysubstance abusers with concurrent alcohol abuse. J Int Neuropsychol Soc 1998; 4(4):319-328.

32. O'Malley S, Adamse M, Heaton R, Gawin FH. Neuropsychological impairment in chronic cocaine abusers. Am Drug Alcohol Abuse 1992; 18:131-144.

33. Strickland TL, Mena I, Villanueva-Meyer J, Miller BL, Cummings J, Mehringer CM, Satz P, Meyers H. Cerebral perfusion and neuropsychological consequences of chronic cocaine use. J Neuropsychiatry Clin Neurosci 1993; 5:419-427.

34. Easton C, Bauer LO. Neuropsychological correlates of urine toxicology results. Prog Neuro-Psychopharmacol Biol Psychiatry 1996; 20(6):969982.

35. Lavie P. The 24-hour sleep propensity function: practical and theoretical implications. In: Monk TH, ed. Sleep, Sleepiness and Performance. Chichester: John Wiley \& Sons, Ltd., 1991:65-93.

36. Roberts DC, Brebner K, Vincler M, Lynch WJ. Patterns of cocaine selfadministration in rats produced by various access conditions under a discrete trials procedure. Drug Alcohol Depend 2002; 67(3):291-299.

37. Erickson TB, Lee J, Zautcke JL, Morris R. Analysis of cocaine chronotoxicology in an urban ED. Am J Emerg Med 1998; 16(6):568571.

38. Giorgetti M, Zhdanova IV. Chronic cocaine treatment induces dysregulation in the circadian pattern of rats' feeding behavior. Brain Res 2000; 877(2):170-175.

39. Johanson CE, Roehrs T, Schuh K, Warbasse L. The effects of cocaine 
on mood and sleep in cocaine dependent males. Exp Clin Psychopharmacol 1999; 7:338-346.

40. Kowatch RA, Schnoll SS, Knisely JS, Green D, Elswick RK. Electroencephalographic sleep and mood during cocaine withdrawal. J Addict Dis 1992; 11:21-45.

41. Van Dongen HP, Maislin G, Mullington JM, Dinges DF. The cumulative cost of additional wakefulness: dose-response effects on neurobehavioral functions and sleep physiology from chronic sleep restriction and total sleep deprivation. Sleep 2003; 26(2):117-126.

42. Harrison Y, Horne JA. The impact of sleep deprivation on decision making: a review. J Exp Psychol, Appl 2000; 6(3):236-249.

43. Maquet P, Smith C, Stickgold R, eds. Sleep and Plasticity. New York: Oxford University Press, 2003.

44. Bradberry $\mathrm{CW}$. Acute and chronic dopamine dynamics in a nonhuman primate model of recreational cocaine use. J Neurosci 2000; 20(18):7109_ 7115.

45. Baumann MH, Becketts KM, Rothman RB. Evidence for alterations in presynaptic serotonergic function during withdrawal from chronic cocaine in rats. Eur J Pharmacol 1995; 282(1-3):87-93.

46. Belej T, Manji D, Sioutis S, Barros HM, Nobrega JN. Changes in serotonin and norepinephrine uptake sites after chronic cocaine: pre- vs. post-withdrawal effects. Brain Res 1996; 736(1-2):287-296.

47. Wilson JM, Carroll ME, Lac ST, DiStefano LM, Kish SJ. Choline acetyltransferase activity is reduced in rat nucleus accumbens after unlimited access to self-administration of cocaine. Neurosci Lett 1994; 180(1):29-32.

48. Kalivas PW, McFarland K, Bowers S, Szumlinski K, Xi ZX, Baker D. Glutamate transmission and addiction to cocaine. Ann NY Acad Sci 2003; 1003:169-175.

49. Xi ZX, Ramamoorthy S, Shen H, Lake R, Samuvel DJ, Kalivas PW. GABA transmission in the nucleus accumbens is altered after withdrawal from repeated cocaine. J Neurosci 2003; 23(8):3498-3505.

50. Volkow ND, Fowler JS, Wang GJ, Hitzemann R, Logan J, Schlyer DJ, Dewey SL, Wolf AP. Decreased dopamine D2 receptor availability is associated with reduced frontal metabolism in cocaine abusers. Synapse 1993; 14(2):169-177.

51. Fleckenstein AE, Gibb JW, Hanson GR. Differential effects of stimulants on monoaminergic transporters: pharmacological consequences and implications for neurotoxicity. Eur J Pharmacol 2000; 406:1-13.

52. Staley JK, Talbot JZ, Ciliax BJ, Miller GW, Levey AI, Kung MP, Kung HF, Mash DC. Radioligand binding and immunoautoradiographic evidence for a lack of toxicity to dopaminergic nerve terminals in human cocaine overdose victims. Brain Res 1997; 747(2):219-229.

53. Kish SJ, Kalasinsky KS, Furukawa Y, Guttman M, Ang L, Li L, Adams 
V, Reiber G, Anthony RA, Anderson W, Smialek J, DiStefano L. Brain choline acetyltransferase activity in chronic, human users of cocaine, methamphetamine, and heroin. Mol Psychiatry 1999; 4(1):26-32.

54. Pace-Schott EF, Morgan P, Edgar C, Malison RT, Hart CL, Walker M, Hobson JA, Stickgold R. Cocaine users differ from normals on cognitive tasks which show declining performance during drug abstinence. Sleep 2004; 27(Supplement):A346.

55. Jones S, Jensen NO, Oliver S, Wesnes KA. First in man cognitive effects of NS2330, a novel monoamine reuptake inhibitor, in volunteers. J Psychopharmacol 1999; 13(Supplement):A26.

56. Teichner G, Homer MD, Harvey RT. Neuropsychological predictors of the attainment of treatment objectives in substance abuse patients. Int Neurosci 2001; 106:253-263.

57. Teichner G, Homer MD, Roitzsch JC, Herron J, Thevos A. Substance abuse treatment outcomes for cognitively impaired and intact outpatients. Addict Behav 2002; 27:751-763. 